\title{
ARTICLE
}

\section{A de novo mutation of the $M Y H 7$ gene in a large Chinese family with autosomal dominant myopathy}

Tetsuya Oda ${ }^{1,7}$, Hui Xiong ${ }^{2,7}$, Kazuhiro Kobayashi ${ }^{1}$, Shuo Wang ${ }^{2}$, Wataru Satake ${ }^{1}$, Hui Jiao ${ }^{2}$, Yanling Yang ${ }^{2}$, Pei-Chieng Cha ${ }^{1}$, Yukiko K Hayashi ${ }^{3}$, Ichizo Nishino ${ }^{4}$, Yutaka Suzuki ${ }^{5}$, Sumio Sugano ${ }^{6}$, Xiru $\mathrm{Wu}^{2}$ and Tatsushi Toda ${ }^{1}$

Laing distal myopathy (LDM) is an autosomal dominant myopathy that is caused by mutations in the slow/beta cardiac myosin heavy-chain (MYH7) gene. It has been recently reported that LDM presents with a wide range of clinical manifestations. We herein report a large Chinese family with autosomal dominant myopathy. The affected individuals in the family presented with foot drop in early childhood, along with progressive distal and proximal limb weakness. Their characteristic symptoms include scapular winging and scoliosis in the early disease phase and impairment of ambulation in the advanced phase. Although limb-girdle muscle dystrophy (LGMD) was suspected initially, a definite diagnosis could not be reached. As such, we performed linkage analysis and detected four linkage regions, namely 1q23.2-24.1, 14q11.2-12, 15q26.2-26.3 and 17q24.3. Through subsequent whole exome sequencing, we found a de novo p.K1617del causative mutation in the MYH7 gene and diagnosed the disease as LDM. This is the first LDM case in China. Our patients have severe clinical manifestations that mimic LGMD in comparison with the patients with the same mutation reported elsewhere.

Human Genome Variation (2015) 2, 15022; doi:10.1038/hgv.2015.22; published online 16 July 2015

\section{INTRODUCTION}

Distal myopathy is characterized by weakness and atrophy of distal limb muscles and is genetically heterogeneous. ${ }^{1}$ Slow/beta cardiac myosin heavy-chain (MYH7) gene is one of the causative genes. Mutations in the MYH7 gene are related to some muscle diseases, including familial hypertrophic or dilated cardiomyopathy, ${ }^{2,3}$ Laing distal myopathy (LDM) ${ }^{4}$ and myosin storage myopathy. ${ }^{5}$ The most common clinical manifestation of LDM is slowly progressive distal limb-muscle weakness, especially in the tibialis anterior and toe extensors muscles, with an early childhood onset. The disease is caused by mutations in the MYH7 gene, which is mainly located in the region encoding the mid-rod domain of the myosin heavy chain. $^{6-8}$ Recently, it has been described in some reports that LDM shows wide variations of clinical manifestations. ${ }^{9-12}$

In this study, we analyzed a five-generation Chinese family with suspected limb-girdle muscle dystrophy (LGMD) using linkage analysis and whole exome sequencing to detect pathogenic mutation in the affected individuals. We found that the patients in the family carry a 3-bp in-frame deletion in the MYH7 gene, p.K1617del, which has been known to be associated with LDM. Their clinical presentations were severe and progressed fast as compared to those with the same mutation that had been previously reported. ${ }^{4,13-16}$ The family is the first LDM case in China and supports a wide variety of clinical presentations of the disease.

\section{MATERIALS AND METHODS}

Samples

The large Chinese family investigated in this study consists of 5 generations and 52 members, including 11 unrelated spouses (Figure 1).
Fourteen family members are affected with this disease. Informed consent was obtained from all participants, and blood samples were collected from 18 family members who agreed to undergo genetic testing. The clinical and examination findings of 14 patients are summarized in Table 1. This study has been approved by the Human Ethics Review Committees of Peking University First Hospital, Kobe University Graduate School of Medicine, and the University of Tokyo.

\section{Sanger sequencing analysis}

Genomic DNA was extracted from whole peripheral blood leukocytes. The MYH7 and HSPA6 genes were amplified by using TaKaRa Ex Taq polymerase (Takara Bio, Shiga, Japan) and KOD Plus Ver. 2 DNA polymerase (TOYOBO, Osaka, Japan), respectively, according to the corresponding manufacturers' instructions. PCR products were direct-sequenced on the 3730xI DNA analyzer (Applied Biosystems, Life Technologies Corporation, Carlsbad, CA, USA) after purification by Wizard SV Gel and PCR Clean-Up System (Promega, Madison, WI, USA). Primer sequences for PCR and sequencing of the MYH7 and HSPA6 genes are available on request.

\section{Microsatellite marker analysis}

PCR of microsatellite markers was carried out using TaKaRa Ex Taq polymerase. Genotypes of the microsatellite markers were determined by using Gene Mapper v4.1 on the 3730xI DNA analyzer.

Single nucleotide polymorphism (SNP) array analysis

SNP genotyping was performed by using GeneChip Mapping $250 \mathrm{~K}$ STY Array (Affymetrix, Santa Clara, CA, USA). SNP genotype calls were generated using the BRLMM algorithm in Affymetrix Genotyping Consol v4.0 application.

\footnotetext{
${ }^{1}$ Division of Neurology/Molecular Brain Science, Kobe University Graduate School of Medicine, Kobe, Japan; ${ }^{2}$ Department of Pediatrics, Peking University First Hospital, Beijing, China; ${ }^{3}$ Department of Pathophysiology, Tokyo Medical University, Tokyo, Japan; ${ }^{4}$ Department of Neuromuscular Research, National Institute of Neuroscience, National Center of Neurology and Psychiatry, Tokyo, Japan; ${ }^{5}$ Department of Computational Biology, Graduate School of Frontier Sciences, the University of Tokyo, Chiba, Japan and ${ }^{6}$ Laboratory of Functional Genomics, Department of Medical Genome Sciences, Graduate School of Frontier Sciences, the University of Tokyo, Tokyo, Japan.

Correspondence: T Toda (toda@med.kobe-u.ac.jp)

${ }^{7}$ These authors contributed equally to this work.

Received 21 January 2015; revised 24 April 2015; accepted 11 May 2015
} 


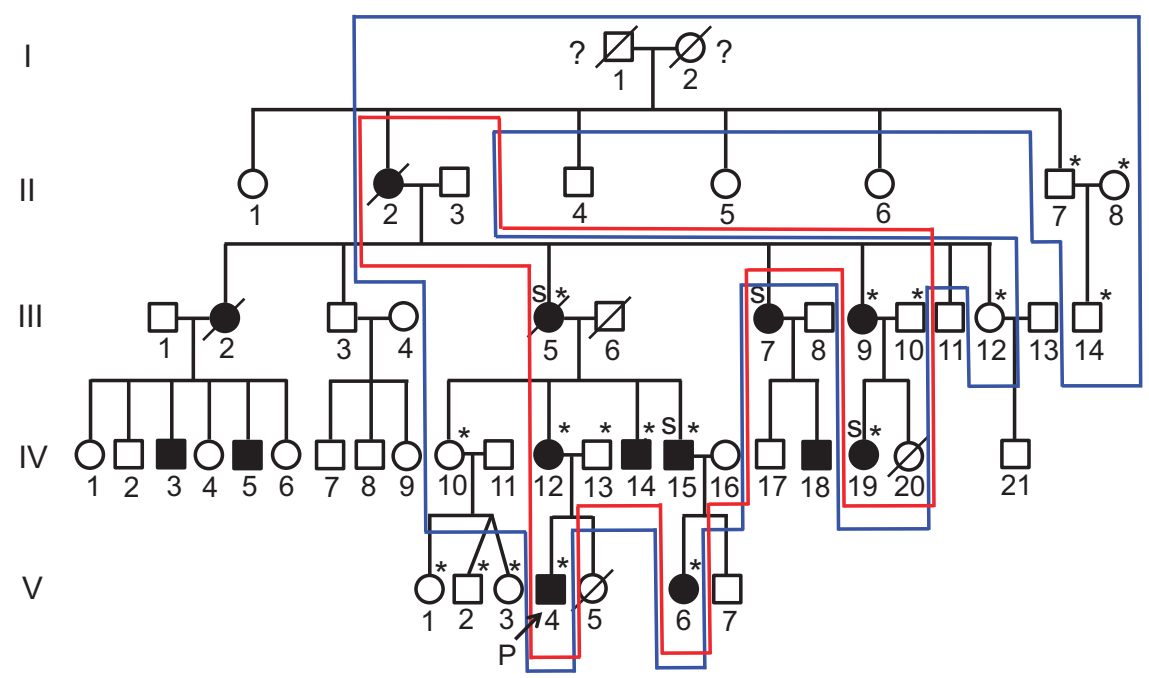

Figure 1. Family pedigree of a Chinese large family with LDM. Asterisk indicates the member whose DNA sample was available. The letter $\mathrm{S}$ indicates a patient with severe scoliosis. Question mark indicates individual whose disease status (affected or unaffected) was unclear. The F1 member set consisting of only affected individuals is surrounded by a red line, and the F2 set consisting of affected and unaffected individuals is surrounded by a blue line.

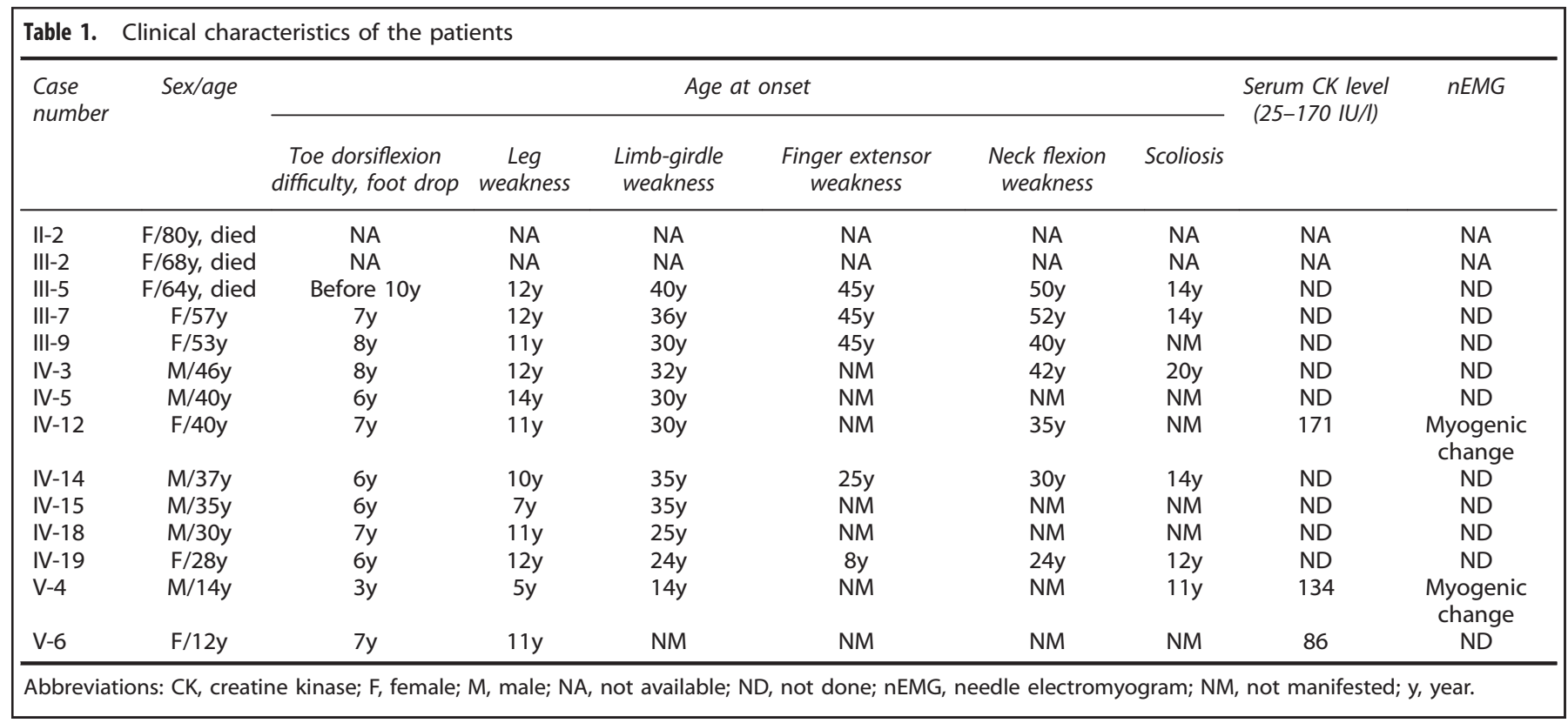

\section{Linkage analysis}

The SNP High Throughput Linkage analysis system v1.5.1 (SNP HiTLink) was used for linkage analysis. ${ }^{17}$ Parametric multipoint linkage analysis was performed using Merlin v1.1.2 program in SNP HiTLink, under the assumptions of autosomal dominant inheritance, complete penetrance and a disease allele frequency of $0.001 .^{18}$ SNP genotype data were filtered based on the following threshold values: call rate of control samples $>0.95, P$ value of Hardy-Weinberg equilibrium test $>0.05$, minimum minor allele frequencies of control samples $=>0.2$, linkage disequilibrium settings of $D^{\prime}=<0.2$ and $r^{2}=<0.2$, minimum interval $=5,000 \mathrm{bp}$, and confidence score of all family members $<0.03$. Logarithm of odds (LOD) scores were calculated. Haplotype prediction was implemented by Allegro v2.0 program ${ }^{19,20}$ in SNP HiTLink.

\section{Exome sequencing}

DNA from the affected individuals (IV-14, IV-19, V-4 and V-6) was enriched for the human exome using Sure Select Human All Exon V4 kit according to the manufacturer's protocol (Agilent Technologies, Santa Clara, CA, USA). The enriched DNAs were sequenced on the HiSeq 2000 system (Illumina, San Diego, CA, USA) with 100-bp paired-end runs. The reads were aligned to the UCSC human reference genome (version hg19) with BWA (Burrows-Wheeler Aligner). Variants, including single nucleotide variations (SNVs), insertions and deletions (indels) were labeled using SAM tools (Sequence Alignment/Map Tools) and GATK (The Genome Analysis Toolkit). SNPs deposited in the 1000 Genomes project data or in the dbSNP (build 132) were removed. We searched for heterozygous SNVs and indels shared by the four patients, but not shared by our in-house disease control SNV database (exome sequencing data from 166 Japanese samples, unpublished data). The indels that were not described as pathogenic in the dbSNP (build 137) were excluded. Afterwards, we focused on the SNVs and indels that were located within the linkage regions. Sanger sequencing confirmed the presence of the two most likely candidates after the aforementioned filtering, which resided in the MYH7 and HSPA6 genes. 

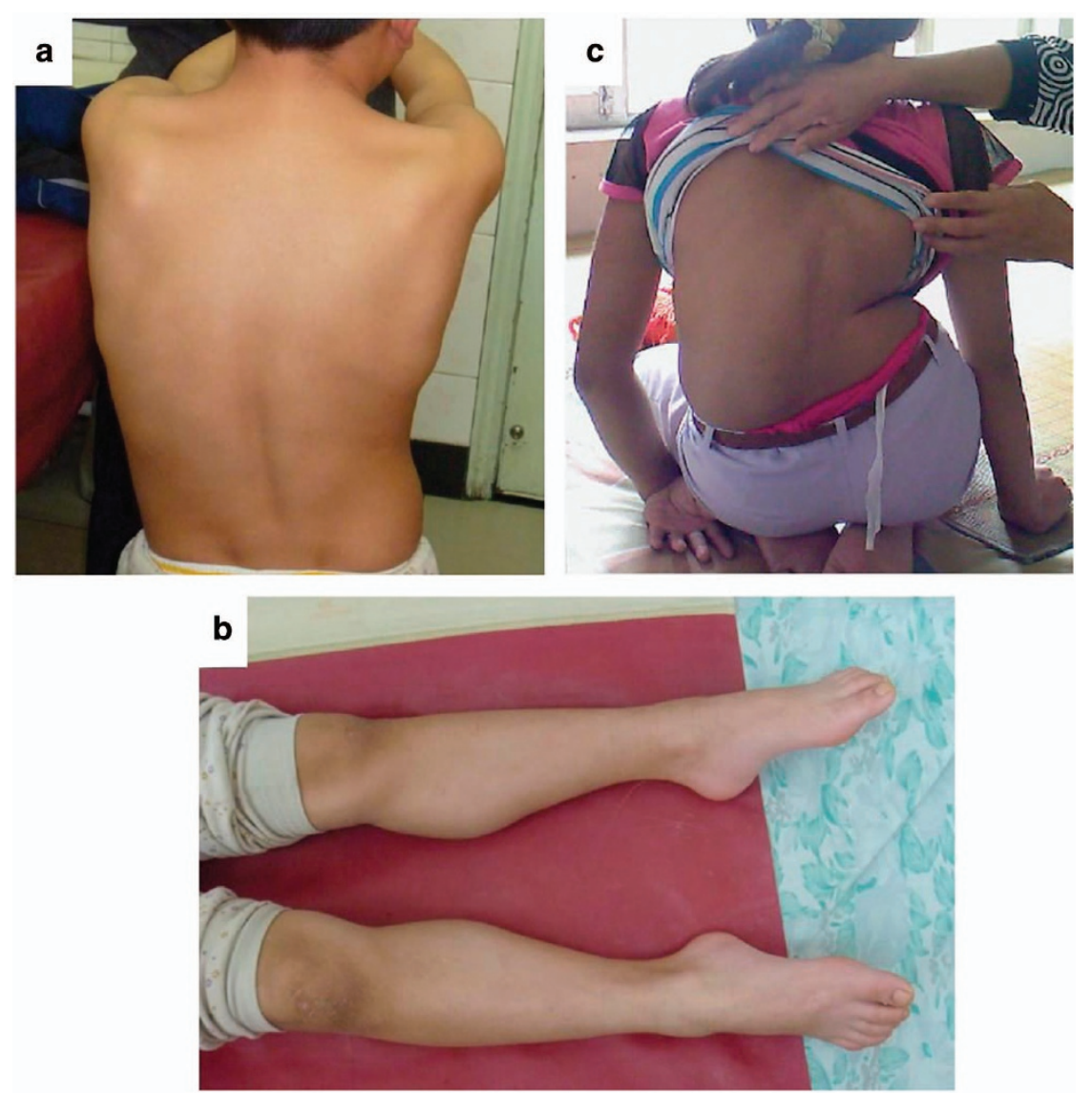

Figure 2. Clinical features of the patients in the family. (a) Mildly winged scapula and scoliosis were present in the V-4 patient. (b) Foot drop and calf muscle hypertrophy were present in the V-4 patient. (c) Severe scoliosis was present in the IV-19 patient.

\section{RESULTS}

Clinical features

The large Chinese family consists of five generations with an autosomal dominant myopathy characterized by onset in early childhood, foot drop, limb-girdle weakness, scoliosis and slow progression (Figure 1). Fourteen patients in the family had obvious muscular weakness and atrophy. Clinical examination and neurophysiological testing were conducted on the patients (Table 1). The core clinical phenotypes of their disorder are described below. They were asymptomatic at birth and developed normally in infancy. The age of onset for the disease was between 3 and 10 years, with the first symptoms being toe dorsiflexion difficulty and foot drop. A few years later, most of the symptomatic individuals showed proximal limb weakness, and some developed mild scapular winging, and/or scoliosis. All of them presented calf muscle hypertrophy (Figure 2a,b). Characteristic features included slowly progressive muscle weakness and atrophy affecting the distal and proximal limbs and girdle. V- 6 was considered to be too young to show this phenotype. Their deep tendon reflexes were decreased. They had positive Gower's sign. They had weakness of finger extensor and neck flexion by the age of 50. Their symptoms were relatively symmetric. There was no gender difference in disease severity. They showed no dysarthria, dysphagia or facial muscle involvement. They also did not present with high arched palate, tremors, sensory disturbances, contractures of joints, respiratory failures or obvious cardiac failures. Their intelligence was normal.

Six patients had sclerosis, and four of them, namely, III-5, III-7, IV-14 and IV-19, showed severe scoliosis (Figure 2c). As a result, their movements were restricted from young adulthood. The patients at $60-70$ years of age in the advanced stage either had an inability to walk without aids or could not work. Patients II-2, III-2 and III-5 died at 64-80 years; their lifespans were almost the same as the unaffected family members. Serum creatine kinase level was almost normal (IV-12, V-4 and V-6). Nerve conduction study revealed normal conduction velocity, whereas concentric needle electromyography revealed myogenic changes in patients IV-12 and V-4. Echocardiogram showed normal results (IV-12, V-4 and V-6). We could not obtain agreements for muscle Computed Tomography (CT), muscle Magnetic Resonance Imaging (MRI) or muscle biopsy from the patients.

\section{Linkage analysis using SNP array data}

This disorder was initially thought to be LGMD1 with childhood onset because it showed autosomal dominant inheritance pattern in the family and the most affected members developed limbgirdle muscle weakness. However, we did not reach a definite diagnosis. Subsequently, we performed genome-wide SNP genotyping for 15 members with blood relationship by Affymetrix GeneChip microarray (QC call rates $>0.96$ ), processed the genotyping data by SNP HiTLink, and performed parametric multipoint linkage analysis by Merlin. To avoid data processing errors, two member sets, namely, F1, consisting of affected individuals only, and F2, consisting of both affected and unaffected individuals, were constructed (Figure 1). The phenotypes of I-1 and I-2 were defined as unknown. When the F1 set was used, the disease locus was mapped to 4 regions on chromosomes $1 q 23.2-24.1,14 q 11.2-12,15 q 26.2-26.3$ and 17q24.3. These loci have LOD scores $>0$ and peak LOD scores $>1$ (maximum LOD scores of 1.81 at $\theta=0$ between rs4492615 and rs12121870 on chromosome 1, between rs10129436 and rs1951061 on chromosome 14, and between rs11634785 and rs4965817 on 


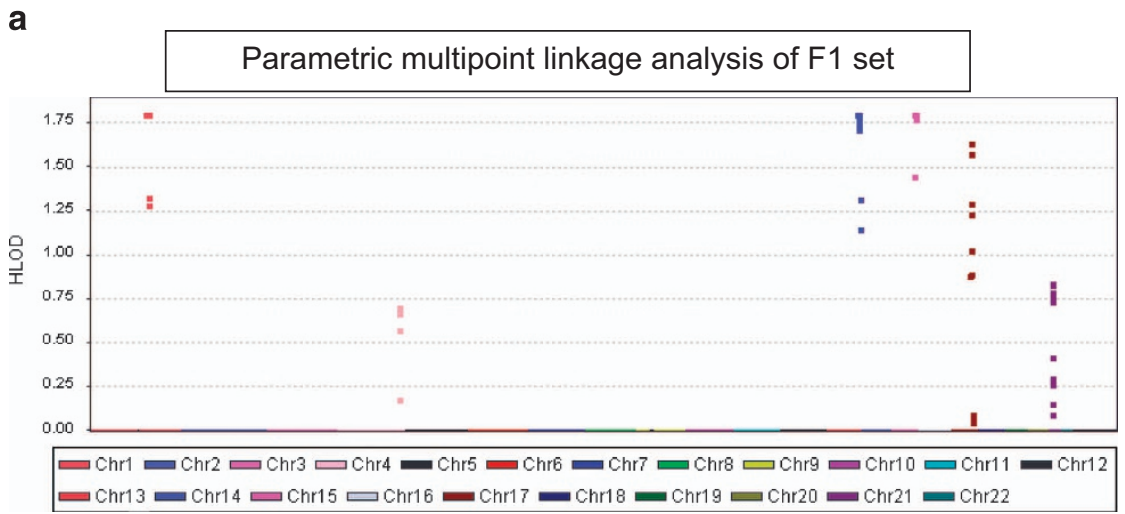

b

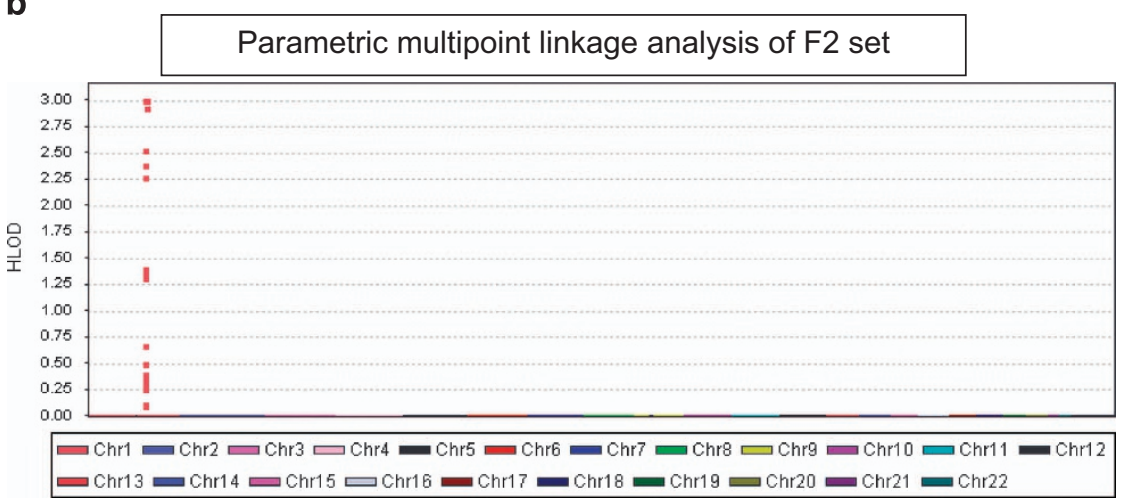

Figure 3. Parametric multipoint linkage analysis of the Chinese family. (a) Linkage analysis of the F1 set mapped the disease locus to 4 regions on chromosomes 1, 14, 15 and 17 with peak LOD scores $>1$. (b) Linkage analysis of the F2 set mapped the disease locus only to chromosome 1. Each dot shows the LOD score at each SNP.

Table 2. Filtering the called variants by exome sequencing

\begin{tabular}{|c|c|c|c|c|}
\hline & IV-14 & IV-19 & $V-4$ & $V-6$ \\
\hline \multicolumn{5}{|l|}{ SNVs } \\
\hline Called SNVs & $\begin{array}{c}150,251 \\
\downarrow\end{array}$ & $\begin{array}{c}172,375 \\
\downarrow\end{array}$ & $\begin{array}{c}231,744 \\
\downarrow\end{array}$ & $\begin{array}{c}262,960 \\
\downarrow\end{array}$ \\
\hline And not in the dbSNP 132 & 7,485 & 10,607 & 14,995 & 12,969 \\
\hline And shared by all four individuals & \multicolumn{4}{|c|}{$\begin{array}{c}194 \\
\downarrow\end{array}$} \\
\hline And located within the F1 linkage regions (chr1, chr14, chr15 and chr17) & \multicolumn{4}{|c|}{$\downarrow$} \\
\hline And located within the F2 linkage region (chr1q23.3-24.1) & \multicolumn{4}{|c|}{1} \\
\hline \multicolumn{5}{|l|}{ INDELS } \\
\hline Called INDELS & 62,860 & 60,479 & 58,671 & 75,259 \\
\hline And located within the F1 linkage regions (chr1, chr14, chr15 and chr17) & \multicolumn{3}{|c|}{21} & $\downarrow$ \\
\hline And located within the F2 linkage region (chr1q23.3-24.1) & \multicolumn{4}{|c|}{4} \\
\hline
\end{tabular}

chromosome 15 , and a maximum LOD score of 1.64 at $\theta=0$ at rs6501530 on chromosome 17; Figure 3a). On the other hand, when the F2 set was used, the disease locus was mapped only to chromosome 1q23.3-24.1 with a LOD score $>0$ (a maximum LOD score of 3.01 at $\theta=0$ between rs7544972 and rs10494458 on chromosome 1; Figure 3b). There were no known LGMD1 loci overlapping these regions. Therefore, we focused on these four genomic regions of $\sim 21 \mathrm{Mb}$ in total as novel LGMD1 locus candidates, especially on the region of 1q23.3-24.1, where the linkage regions of the F1 and F2 sets overlapped. 
Whole exome sequencing

We checked if the 4 linkage regions contained any copy number variations (CNVs) that are likely to be pathogenic using SNP genotyping data and found none. Subsequently, whole exome sequencing was performed for four affected members of the family (IV-14, IV-19, V-4 and V-6). At first, we found only one heterozygous SNV that was shared by the four patients within the linkage region of 1q23.3-24.1 (Table 2). It is a novel nonsynonymous SNV in the HSPA6 (heat-shock 70-kDa protein 6) gene on 1q23.3, involving a c.661G $>$ A nucleotide change and resulting in a p.V221M amino-acid substitution in the HSPA6 protein. This SNV was estimated to be 'probably damaging', 'damaging' and 'deleterious' by the PolyPhen-2, SIFT and PROVEAN softwares, respectively, and was not in our in-house SNV database. Next, this heterozygous C.661G $>$ A change of the HSPA6 gene was further investigated in the 18 family members who provided blood samples by Sanger sequencing. All the affected members and, strangely, one unaffected old member (II-7) shared this change. We carefully checked the HSPA6 gene and found that the gene is positioned at the boundary of the strong linkage region, where the LOD score was decreasing in the F2 set. Haplotype analyses using both SNP and microsatellite genotyping data revealed that the unaffected member (II-7) shared a part of the haplotype with all the affected members on 1q23.1-23.3 where c.661G $>$ A of HSPA6 possibly resides (Supplementary Figures S1 and S2). Therefore, we concluded that the c.661G $>$ A change was not a disease-causing mutation but a private mutation in the family and excluded the HSPA6 gene from the list of candidate genes. Because we did not find any other heterozygous SNVs in the 1q23.2-24.1 region, we decided to search for mutation in the three other linkage regions, namely, $14 q 11.2-12,15 q 26.2-26.3$ and 17q24.3. By doing so, we found six additional heterozygous SNVs that were shared by all of the four patients, adding up to a total of seven SNVs in the linkage regions from the F1 set (Table 2, upper panel). Three out of the six additional SNVs are located in exons or around splice sites. One of these three SNVs is a non-synonymous SNV, which exists in the TRAV29 gene that is mainly expressed in T cells. Therefore, we concluded that the seven SNVs were not pathogenic.

On the other hand, we mapped indels separately from the aforementioned SNVs. We found 21 heterozygous indels that were shared by all 4 patients within the 4 linkage regions of the F1 set (Table 2, lower panel). Among the 21 indels, we noticed a 3-bp in-frame deletion in the MYH7 gene on 14q11.2, c.4849-4851del, which results in a p.K1617del amino-acid deletion. This heterozygous c.4849-4851 del of the MYH7 gene was further investigated by Sanger sequencing in the 18 family members with available blood samples (Figure 4). This deletion was confirmed to clearly segregate with the disease. The deletion has been known as a pathogenic mutation that causes LDM (rs121913648). The 20 other indels were all found to have been deposited in the dbSNP (build 137) and have not been described as pathogenic. Therefore, we concluded that c.4849-4851del of the MYH7 gene is the causative mutation of this LGMD1-like myopathy in the Chinese family and made a definite diagnosis of the disease as LDM. Haplotype analyses using both SNP and microsatellite genotyping data revealed that the unaffected members (II-7 and III-14) have a haplotype in common with all affected members on 14q11.2, where the c.4849-4851 del of MYH7 should reside (Supplementary Figures S3 and S4). However, the two members II-7 and III-14 did not carry the deletion. Because I-1 and I-2 in the pedigree seemed to have neither subjective nor objective symptoms according to the family history, they might be unaffected. Therefore, it is highly probable that the deletion mutation of the MYH7 gene in our cases has occurred de novo during transmission from the first generation to the II- 2 affected individual. This is possibly why the linkage region of $14 q 11.2$ was detected from the F1 set, where the $\mathrm{MYH7}$ gene exists, but undetectable by linkage analysis of the F2 set.

\section{DISCUSSION}

The MYH7 gene, encoding the slow/beta cardiac myosin heavy chain, is known as the cause of hypertrophic or dilated cardiomyopathy, ${ }^{2,3} \mathrm{LDM}^{4}$ and myosin storage myopathy. ${ }^{5}$ Thus far, more than 200 dominant mutations in MYH7 have been reported to be associated with cardiomyopathy, but only a small number of them cause skeletal myopathy. Almost all of them have been found in Caucasians. ${ }^{7,8}$ In Asian people, although many MYH7 mutations have been reported for cardiomyopathy previously, ${ }^{21,22}$ only one Korean familial case with LDM was reported to carry a mutation in that gene. ${ }^{12}$ Here, we describe the clinical and genetic research of a large Chinese family with autosomal dominant myopathy. By genetic analysis of the family, a pathogenic in-frame deletion mutation, p.K1617del, in the MYH7 gene was detected. To our knowledge, this is the first report of LDM caused by MYH7 mutation in Chinese and the second in an Asian population.

At first, we suspected that our patients had mimic symptoms of LGMD1 with childhood onset. Recently, the expanded clinical spectrum of LDM caused by MYH7 mutations has been described. ${ }^{9-12}$ For example, age of disease onset varies, as there is a wide range from birth to 50 years of age. Distribution and severity of muscle weakness also vary. Interestingly, some reports showed clinical heterogeneity in patients with the same mutations in the MYH7 gene. ${ }^{9,11,23}$ Several cases with p.K1617del mutation in the MYH7 gene, the same as described in the present study, have been reported previously. ${ }^{4,16}$ For instance, an Austrian family with

\section{$\frac{L}{C T G} \frac{R}{A G G} \frac{V}{G T G} \frac{K}{A A G} \frac{K}{A A G} \frac{K / M}{A A G} \frac{M / E}{A T G G / G} \frac{G / D}{D A} \frac{D / L}{\text { A A G }}$ \\ Affected \\ C T G A G G T G A A G A G A T G G A A G G G A C C T C

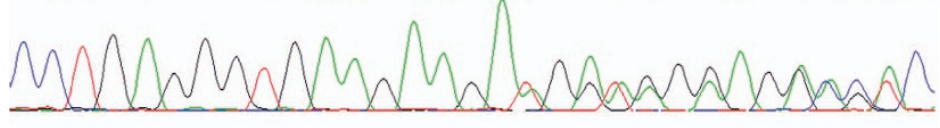 \\ Unaffected $\frac{L}{C T G} \frac{R}{A G G} \frac{V}{G T G} \frac{K}{A A G} \frac{K}{A A G A A G} \frac{K}{A T G G A A} \frac{E}{G G A G A C}$

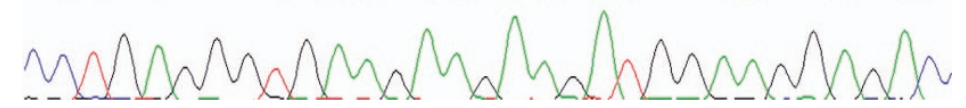

Figure 4. Sanger sequencing analysis of the patients. A heterozygous 3 bp deletion in the MYH7 gene, c.4849-4851del, resulting in p.K1617del, was confirmed. 
the deletion had an onset in the middle teen years, and another German family had an onset in early childhood. ${ }^{4,13,14}$ Their initial symptoms were weakness of toe and ankle dorsiflexion. The clinical courses were slowly progressive, cardiomyopathy did not appear, and serum creatine kinase levels were normal or mildly elevated. Ambulatory activity was retained in the two families. Another sporadic case with onset in early childhood showed weakness of neck flexion, scapular and pectoral muscles at later stages, followed by scoliosis and contracture of joints at the end stage. ${ }^{15}$ A Hungarian Roma family was recently reported. ${ }^{16}$ The propositus showed more severe phenotypes including early loss of ambulation at 27 years and kyphoscoliosis. As for the Chinese family in our study, clinical symptoms were consistent with LDM. However, our cases presented with symptoms of truncal and proximal limb-girdle muscle impairment, such as Gower's sign, scapular winging and scoliosis in the early disease phase. They showed more rapid progression than typical LDM cases and had difficulty walking in advanced stage. Therefore, these additional clinical findings indicate that our cases had a severe form of LDM with p.K1617del mutation in the MYH7 gene and suggest a wide clinical spectrum associated with this deletion mutation.

Although it is still unknown why myopathy associated with MYH7 mutations presents with a wide variety of clinical symptoms, Tasca et al. ${ }^{10}$ suggested that it might be influenced by genetic or environmental modifiers. For example, fiber type proportion in human skeletal muscle is different based on race ${ }^{24}$ and is influenced by both inherited factors and environmental factors. ${ }^{25}$ Our cases showed noticeable symptoms of limb-girdle weakness in the early disease phase and severe scoliosis. A Korean myopathy family with the p.A1439P MYH7 mutation was described to have prominent paraspinal and proximal muscle involvements. $^{12}$ The difference between clinical phenotypes of MYH7 myopathy in Asia and in other ethnic areas might be associated with geographical differences of genetic backgrounds or environmental factors.

We also detected a novel missense substitution, p.V221M, in the HPSA6 gene, which encodes a member of $70-\mathrm{kDa}$ heat-shock protein (HSP70) family. Although most of the function of the HSPA6 protein is still unclear, ${ }^{26}$ it might be possible that the HSPA6 gene is one of the genetic modifiers for the disease and that the missense substitution made the patients in our family relatively severe. HSPA6 is known to form HSP70-heteromeric complexes with HSPA1A and HSPA8, and also form complexes with the $40-\mathrm{kDa}$ heat-shock protein (HSP40) family, which is a cochaperone of the HSP70 family. ${ }^{27}$ The DNAJB6 gene encodes a member (DNAJB6) of the HSP40 family and has been recently identified to be the causative gene for LGMD1D. ${ }^{28-30}$ DNAJB6 was reported to be a co-chaperone of $\mathrm{HSPA}^{31}$ and interact with the chaperone-assisted selective autophagy complex containing HSPA8, BAG3, STUB1 and HSPB8, which is important for Z-disk maintenance. ${ }^{32}$ Mutations in BAG3 cause a myofibrillar myopathy. ${ }^{33}$ Moreover, the HSPA6 p.V221M substitution found in this study affects the highly conserved amino acid within the ATPase domain. ${ }^{34}$ Furthermore, the valine residue is considered to be the most significant site for hydrophobic interaction between the ATPase domain of HSPA6 and J domain of the HSP40 family. ${ }^{35}$ Therefore, it is possible that HSPA6 may bind to DNAJB6 for muscle maintenance and that the p.V221M substitution may alter the binding status of HSPA6, which is not strong enough to be pathogenic but could modify the disease phenotype. However, we could not evaluate this hypothesis, as all of the patients in the investigated family have both the deletion mutation in the MYH7 gene and the substitution in HSPA6 and none of them carry only the MYH7 deletion.

Haplotype analyses indicated that even though the unaffected members II-7 and III-14 have the same haplotype on chromosome $14 \mathrm{q} 11.2$ as the affected members, II-7 and III-14 did not have the p.K1617del mutation in the MYH7 gene. Given that I-1 and I-2 may be unaffected, the finding suggested that the p.K1617del mutation is a de novo mutation in the II-2 affected individual. Careful attention should be paid to de novo mutations; otherwise, they could mislead us in linkage analysis. Considering that the $p$. K1617del mutation found in some families has occurred independently in the world, ${ }^{4,15,16}$ DNA sequences at this site may be naturally vulnerable to deletion, just like the p.E1508del mutation in the MYH7 gene. ${ }^{36}$

Myopathy, including distal myopathy, is a clinically and genetically heterogeneous disease. For diagnosis of myopathy, it is helpful for physicians to take advantage of information from muscle CT, muscle MRI and/or muscle biopsy, in addition to detailed examinations of clinical manifestations. Although we could not obtain the patients' consent for these clinical examinations, a combined approach of linkage analysis and whole exome sequencing had allowed us to make a definite diagnosis of their myopathy as LDM caused by the MYH7 mutation. Although numerous causative genes for myopathy are known so far, the number of identified genes will certainly increase in the future. New technologic genetic testing, such as next generation sequencing, is a very useful and powerful tool for diagnosis of heterogeneous disease, even if clinical information is limited. In addition, we suggest that as MYH7 myopathy has a variety of symptoms, genetic tests for MYH7 mutations should be performed for undiagnosed inherited or sporadic cases with various symptoms of distal myopathy. Further analysis of myopathy is needed to clarify and classify its pathogenic mechanisms.

\section{ACKNOWLEDGEMENTS}

The authors thank all members of the Chinese family in this study. We thank Tatsuya Nishioka (Kobe University Graduate School of Medicine), Kiyomi Imamura and Fusano Todokoro (Tokyo University Graduate School of Frontier Sciences) for their cooperation in whole exome sequencing analysis. We thank Yuko Ando (Kobe University Graduate School of Medicine) for her cooperation in Sanger sequencing and whole exome sequencing analyses. This work was supported by the Grant-in-Aid for Scientific Research on Innovative Areas (22129006) from the Ministry of Education, Culture, Sports, Science and Technology of Japan, and the Intramural Research Grant for Neurological and Psychiatric Disorders of National Center of Neurology and Psychiatry (26-8) from the Ministry of Health, Labor and Welfare of Japan. This work was also supported by grants from the National Natural Science Foundation of China (81271400), and the National Basic Research Program of China (2012CB944602).

\section{COMPETING INTERESTS}

The authors declare no conflict of interest.

\section{REFERENCES}

1 Udd B. Distal myopathies - New genetic entities expand diagnostic challenge. Neuromuscul Disord 2012; 22: 5-12.

2 Geisterfer-Lowrance AAT, Kass S, Tanigawa G, Vosberg H-P, McKenna W, Seidman CE et al. A molecular basis for familial hypertrophic cardiomyopathy: a $\beta$ cardiac myosin heavy chain gene missense mutation. Cell 1990; 62: 999-1006.

3 Kamisago M, Sharma SD, DePalma SR, Solomon S, Sharma P, McDonough B et al. Mutations in sarcomere protein genes as a cause of dilated cardiomyopathy. N Engl J Med 2000; 343: 1688-1696.

4 Meredith C, Herrmann R, Parry C, Liyanage K, Dye DE, Durling HJ et al. Mutations in the slow skeletal muscle fiber myosin heavy chain gene (MYH7) cause Laing early-onset distal myopathy (MPD1). Am J Hum Genet 2004; 75: 703-708.

5 Tajsharghi H, Thornell L-E, Lindberg C, Lindvall B, Henriksson K-G, Oldfors A. Myosin storage myopathy associated with a heterozygous missense mutation in MYH7. Ann Neurol 2003; 54: 494-500.

6 Laing NG, Laing BA, Meredith C, Wilton SD, Robbins $\mathrm{P}$, Honeyman $\mathrm{K}$ et al. Autosomal dominant distal myopathy: linkage to chromosome 14. Am J Hum Genet 1995; 56: 422-427.

7 Oldfors A. Hereditary myosin myopathies. Neuromuscul Disord 2007; 17: 355-367.

8 Tajsharghi $\mathrm{H}$, Oldfors A. Myosinopathies: pathology and mechanisms. Acta Neuropathol 2013; 125: 3-18. 
9 Muelas N, Hackman P, Luque H, Garcés-Sánchez M, Azorín I, Suominen T et al. MYH7 gene tail mutation causing myopathic profiles beyond Laing distal myopathy. Neurology 2010; 75: 732-741.

10 Tasca G, Ricci E, Penttilä S, Monforte M, Giglio V, Ottaviani P et al. New phenotype and pathology features in MYH7-related distal myopathy. Neuromuscul Disord 2012; 22: 640-647.

11 Clarke NF, Amburgey K, Teener J, Camelo-Piragua S, Kesari A, Punetha J et al. A novel mutation expands the genetic and clinical spectrum of MYH7-related myopathies. Neuromuscul Disord 2013; 23: 432-436.

12 Park J-M, Kim YJ, Yoo JH, Hong YB, Park JH, Koo H et al. A novel MYH7 mutation with prominent paraspinal and proximal muscle involvement. Neuromuscul Disord 2013; 23: 580-586.

13 Zimprich F, Djamshidian A, Hainfellner JA, Budka H, Zeitlhofer J. An autosomal dominant early adult-onset distal muscular dystrophy. Muscle Nerve 2000; 23: 1876-1879.

14 Voit T, Kutz P, Leube B, Neuen-Jacob E, Schröder JM, Cavallotti D et al. Autosomal dominant distal myopathy: further evidence of a chromosome 14 locus. Neuromuscul Disord 2001; 11: 11-19.

15 Lamont PJ, Udd B, Mastaglia FL, de Visser M, Hedera P, Voit T et al. Laing early onset distal myopathy: slow myosin defect with variable abnormalities on muscle biopsy. J Neurol Neurosurg Psychiatry 2006; 77: 208-215.

16 Komlósi K, Hadzsiev K, Garbes L, Martínez-Carrera LA, Pál E, Sigurðsson JH et al. Exome sequencing identifies Laing distal myopathy $M Y H 7$ mutation in a Roma family previously diagnosed with distal neuronopathy. Neuromuscul Disord 2014; 24: 156-161.

17 Fukuda Y, Nakahara Y, Date H, Takahashi Y, Goto J, Miyashita A et al. SNP HiTLink: a high-throughput linkage analysis system employing dense SNP data. BMC Bioinformatics 2009; 10: 121 .

18 Abecasis GR, Cherny SS, Cookson WO, Cardon LR. Merlin - rapid analysis of dense genetic maps using sparse gene flow trees. Nat Genet 2002; 30: 97-101.

19 Gudbjartsson DF, Jonasson K, Frigge ML, Kong A. Allegro, a new computer program for multipoint linkage analysis. Nat Genet 2000; 25: 12-13.

20 Gudbjartsson DF, Thorvaldsson T, Kong A, Gunnarsson G, Ingolfsdottir A. Allegro version 2. Nat Genet 2005; 37: 1015-1016.

21 Otsuka H, Arimura T, Abe T, Kawai H, Aizawa Y, Kubo T et al. Prevalence and distribution of sarcomeric gene mutations in Japanese patients with familial hypertrophic cardiomyopathy. Circ J 2012; 76: 453-461.

22 Zou Y, Wang J, Liu X, Wang Y, Chen Y, Sun K et al. Multiple gene mutations, not the type of mutation, are the modifier of left ventricle hypertrophy in patients with hypertrophic cardiomyopathy. Mol Biol Rep 2013; 40: 3969-3976.

23 Uro-Coste E, Arné-Bes M-C, Pellissier J-F, Richard P, Levade T, Heitz F et al. Striking phenotypic variability in two familial cases of myosin storage myopathy with a MYH7 Leu1793Pro mutation. Neuromuscul Disord 2009; 19: 163-166.

24 Ama PF, Simoneau JA, Boulay MR, Serresse O, Theriault G, Bouchard C. Skeletal muscle characteristics in sedentary black and Caucasian males. $J$ Appl Physiol 1986; 61: 1758-1761.
25 Simoneau J-A, Bouchard C. Genetic determinism of fiber type proportion in human skeletal muscle. FASEB J 1995; 9: 1091-1095.

26 Hageman J, van Waarde MAWH, Zylicz A, Walerych D, Kampinga HH. The diverse members of the mammalian HSP70 machine show distinct chaperone-like activities. Biochem J 2011; 435: 127-142.

27 Chow AM, Mok P, Xiao D, Khalouei S, Brown IR. Heteromeric complexes of heat shock protein 70 (HSP70) family members, including Hsp70B', in differentiated human neuronal cells. Cell Stress and Chaperones 2010; 15: 545-553.

28 Kampinga HH, Craig EA. The Hsp70 chaperone machinery: J-proteins as drivers of functional specificity. Nat Rev Mol Cell Biol 2010; 11: 579-592.

29 Harms MB, Sommerville RB, Allred P, Bell S, Ma D, Cooper $\mathrm{P}$ et al. Exome sequencing reveals DNAJB6 mutations in dominantly-inherited myopathy. Ann Neurol 2012; 71: 407-416.

30 Sarparanta J, Jonson PH, Golzio C, Sandell S, Luque H, Screen M et al. Mutations affecting the cytoplasmic functions of the co-chaperone DNAJB6 cause limbgirdle muscular dystrophy. Nat Genet 2012; 44: 450-455.

31 Chuang JZ, Zhou H, Zhu M, Li SH, Li XJ, Sung CH. Characterization of a brainenriched chaperone, MRJ, that inhibits Huntingtin aggregation and toxicity independently. J Biol Chem 2002; 277: 19831-19838.

32 Arndt V, Dick N, Tawo R, Dreiseidler M, Wenzel D, Hesse M et al. Chaperoneassisted selective autophagy is essential for muscle maintenance. Curr Biol 2010; 20: $143-148$.

33 Selcen D, Muntoni F, Burton BK, Pegoraro E, Sewry C, Bite AV et al. Mutation in $B A G 3$ causes severe dominant childhood muscular dystrophy. Ann Neurol 2009; 65: 83-89.

34 Wisniewska M, Karlberg T, Lehtiö L, Johansson I, Kotenyova T, Moche $M$ et al. Crystal structures of the ATPase domains of four human Hsp70 isoforms: HSPA1L/Hsp70-hom, HSPA2/Hsp70-2, HSPA6/Hsp70B', and HSPA5/BiP/GRP78. PLoS One 2010; 5: e8625.

35 Gruschus JM, Greene LE, Eisenberg E, Ferretti JA. Experimentally biased model structure of the Hsc70/auxilin complex: substrate transfer and interdomain structural change. Protein Sci 2004; 13: 2029-2044.

36 Dubourg O, Maisonobe T, Behin A, Suominen T, Raheem O, Penttilä S et al. A novel MYH7 mutation occurring independently in French and Norwegian Laing distal myopathy families and de novo in one Finnish patient. J Neurol 2011; 258: 1157-1163.

(c) $\Theta$ This work is licensed under a Creative Commons AttributionBY NC No NonCommercial-NoDerivs 4.0 International License. The images or other third party material in this article are included in the article's Creative Commons license, unless indicated otherwise in the credit line; if the material is not included under the Creative Commons license, users will need to obtain permission from the license holder to reproduce the material. To view a copy of this license, visit http:// creativecommons.org/licenses/by-nc-nd/4.0/

Supplemental Information for this article can be found on the Human Genome Variation website (http://www.nature.com/hgv). 